\title{
Síndrome nefrótico secundario a glomerulonefritis membranosa asociada al tratamiento con litio.
}

\author{
Mario Fernández-Ruiz ${ }^{{ }^{*}}$, Esther González ${ }^{2}$, Eduardo Hernández ${ }^{2}$, Manuel Praga ${ }^{2}$ \\ ${ }^{1}$ Unidad de Enfermedades Infecciosas, Hospital Universitario "12 de Octubre", Instituto de Investigación Hospital “12 de Octubre" (i+12), Madrid \\ ${ }^{2}$ Servicio de Nefrología, Hospital Universitario "12 de Octubre", Instituto de Investigación Hospital "12 de Octubre" (i+12), Madrid \\ Corresponding authors: Unidad de Enfermedades Infecciosas. Hospital Universitario "12 de Octubre". Centro de Actividades Ambulatorias, planta \\ 2a , bloque D. Avda. de Córdoba, s/n. Código postal: 28041. Madrid. Dirección de correo electrónico: mario_fdezruiz@yahoo.es
}

Palabras Claves: Case report. Carbonato de litio. Glomerulonefritis membranosa. Síndrome nefrótico. Proteinuria. Recibido: 22 de enero 2017; Aceptado: 1 de febrero 2017; Publicado: 3 de febrero 2017.

Copyright: (c) 2017 Authors. This is an open-access article distributed under the terms of the Creative Commons Attribution License, which permits unrestricted use, distribution, and reproduction in any medium, provided the original author and source are credited.

Editor: Alberto M. Borobia

Citar como: Fernández-Ruiz M., González E., Hernández E., Praga M. Síndrome nefrótico secundario a glomerulonefritis membranosa asociada al tratamiento con litio. IBJ Clin Pharmacol 2017 1(1):e0006.

Fuentes de financiación: Mario Fernández Ruiz disfruta de un contrato de investigación clínica "Juan Rodés" (JR14/00036) del Instituto de Salud Carlos III, Ministerio de Economía y Competitividad.

Conflictos de intereses: Los autores declaran que no existen conflictos de intereses en relación con el presente trabajo. 
El litio es un metal alcalino que constituye un tratamiento ampliamente establecido en el trastorno afectivo bipolar desde hace décadas ${ }^{1}$. Su administración ha sido vinculada a diversos efectos adversos a nivel renal: diabetes insípida nefrogénica (el más frecuente), toxicidad aguda (con poliuria y acidosis tubular), nefropatía túbulo-intersticial crónica (con deterioro progresivo del filtrado glomerular), y lesión glomerular directa ${ }^{1-3}$. La aparición de un síndrome nefrótico (SN) supone una complicación más infrecuente, con una primera descripción en la literatura en $1973^{3-13}$. Su sustrato morfológico es variable, como demuestra una revisión de los 19 casos publicados anteriormente a 1997 , con predominio de la enfermedad por mínimos cambios ${ }^{3,4,7-9,11} \mathrm{y}$, en menor medida, glomeruloesclerosis focal y segmentaria ${ }^{6,10}$. Presentamos un inusual ejemplo de SN secundario a glomerulonefritis membranosa (GMN) en un paciente sometido a tratamiento con carbonato de litio, con posterior remisión clínica y analítica del mismo tras la suspensión del fármaco.

Varón de 53 años, con antecedentes de politoxicomanía (alcohol y cocaína inhalada) e infección crónica por virus de la hepatitis $\mathrm{C}$, con datos histológicos de hepatitis periportal sin progresión a cirrosis. Permanecía en seguimiento psiquiátrico con el diagnóstico de trastorno afectivo bipolar y su tratamiento habitual incluía alprazolam, lamotrigina, venlafaxina y mirtazapina, así como carbonato de litio a dosis de $200 \mathrm{mg} / 8$ horas (iniciado cuatro meses antes del ingreso). Éste fue motivado por la aparición de edemas en párpados y miembros inferiores, de varias semanas de evolución, acompañados de dificultad para la concentración, inestabilidad en la deambulación y temblor en manos. La exploración física confirmó la presencia de edemas con fóvea hasta rodillas, así como de un nistagmus horizontal bilateral agotable, mioclonias posturales y rigidez espástica axial con fenómeno de "rueda dentada" en miembros superiores. Analíticamente destacaba la presencia de un SN completo sin deterioro de función renal (proteínas totales 5,7 $\mathrm{g} / \mathrm{dL}$; albúmina 2,47 g/dL; colesterol $275 \mathrm{mg} / \mathrm{dL}$; triglicéridos $596 \mathrm{mg} / \mathrm{dL}$; creatinina $0,6 \mathrm{mg} / \mathrm{dL}$; filtrado glomerular estimado $106 \mathrm{~mL} /$ minuto; proteinuria 9,12 g/día); el sedimento urinario resultó anodino. Los restantes parámetros hematológicos y bioquímicos se encontraban dentro de la normalidad, incluyendo función tiroidea, consumo de complemento y recuento de inmunoglobulinas. Los niveles de litio en sangre fueron de 1 $\mathrm{mEq} / \mathrm{L}$ (rango terapéutico: 0,6 - 1,2 mEq/L). La determinación de crioglobulinas (criocrito $0,5 \%$ ) y de anticuerpos antinucleares (titulación 1/40) fue débilmente positiva, con negatividad del resto del estudio de autoinmunidad. La ecografía abdominal mostró ambos riñones de tamaño y morfología conservados, en tanto que la biopsia renal reveló diez glomérulos de características ópticamente normales; mediante inmunofluorescencia directa se demostró el depósito granular y membranoso de IgG y C3, compatible con el diagnóstico de GMN en estadio I. No fue posible el estudio con microscopía electrónica por ausencia de material valorable. Tras establecer el juicio clínico de toxicidad neurológica y SN secundario a GMN incipiente en relación con la administración de carbonato de litio, se decidió suspender dicho tratamiento. Al cabo de seis semanas el paciente se encontraba asintomático, con remisión completa de los edemas y de la clínica neurológica, y progresiva normalización analítica (proteínas totales $7,3 \mathrm{~g} / \mathrm{dL}$; albúmina 3,4 g/dL; proteinuria 1,68 g/día). La aplicación del algoritmo de Naranjo de evaluación de causalidad en reacciones adversas a medicamentos (RAM) ${ }^{12}$ permitió calificar como "probable" (7 puntos) la implicación del tratamiento con carbonato de litio en el desarrollo de la GMN. Tras dos años y medio de seguimiento persiste la remisión del SN (albúmina 4,35 $\mathrm{g} / \mathrm{dL}$; proteinuria 0,19 g/día). Se procedió a la notificación de la sospecha de RAM al Sistema Español de Farmacovigilancia (número de notificación 13-607547).

El desarrollo de proteinuria leve constituye un evento relativamente común en el transcurso de la terapia con litio $^{3}$. Su evolución hacia un SN secundario a lesión glomerular resulta más infrecuente, si bien dicha complicación aparece bien documentada en la literatura, en la que se describe la rápida desaparición de la proteinuria tras la interrupción del tratamiento ${ }^{5,9}$ y su recidiva en una segunda exposición ${ }^{4,13}$. El mecanismo íntimo de esta asociación permanece mal caracterizado, habiéndose sugerido en la patogenia de la enfermedad por mínimos cambios un fenómeno de lesión podocitaria directa mediada por linfocitos $\mathrm{T}$ y citoquinas (interleuquina $2 \mathrm{y}$ factor de necrosis tumoral $\alpha)^{3,5,8}$. También se ha invocado la posible interacción entre este catión y la carga aniónica de la membrana basal glomerular, resultando en un aumento de su permeabilidad a determinadas macromoléculas como las proteínas plasmáticas. Los niveles de litio permanecieron en rangos terapéuticos en la mayor parte de los casos publicados ${ }^{3,4,7}$. Por otra parte, la excreción renal del litio se encuentra disminuida en los pacientes con $\mathrm{SN}^{9,10}$, circunstancia que puede precipitar de forma concurrente su toxicidad neurológica, como en el ejemplo aportado. La aparición del SN parece ser independiente del tiempo de exposición ${ }^{11}$, aunque frecuentemente tiene lugar dentro del primer año de tratamiento ${ }^{3}$. La demostración de una GMN en este contexto constituye un hallazgo particularmente inusual, y en la citada revisión de Bosquet et $\mathrm{al}^{3}$ sólo se describe esta entidad en dos de los 17 casos de SN con documentación histológica ${ }^{13}$. Kala et $\mathrm{al}^{14}$ han comunicado recientemente el primer caso pediátrico de GMN en un paciente de 13 años sometido a tratamiento con litio durante más de cinco años. El desarrollo de GMN también a sido vinculado a la toxicidad por otros metales alcalinos, como el oro o el mercurio, circunstancia que refuerza dicha aso- 
ciación patogénica ${ }^{14}$. La remisión del SN tiene lugar generalmente en un plazo variable tras la suspensión del carbonato de litio (de dos a seis semanas) sin necesidad de asociar tratamiento esteroideo en la mayor parte de los pacientes ${ }^{3,5,9,10}$, cuya indicación quedaría reservada a la proteinuria persistente o recurrencia del $\mathrm{SN}^{4}$. El caso que presentamos confirma la validez de dicha estrategia conservadora, incluso ante un sustrato anatomopatológico infrecuente en la toxicidad glomerular mediada por litio como es la GMN.

\section{References}

[1] Moncrieff J. Forty years of lithium therapy. Arch Gen Psychiatry. 1997;54:9-13.

[2] Markowitz GS, Radhakrishnan J, Kambham N, Valeri AM, Hines WH, D'Agati VD. Lithium nephrotoxicity: a progressive combined glomerular and tubulointerstitial nephropathy. J Am Soc Nephrol. 2000;11:1439-48.

[3] Bosquet S, Descombes E, Gauthier T, Fellay G, Regamey C. Nephrotic syndrome during lithium therapy. Nephrol Dial Transplant. 1997;12:2728-31.

[4] Richman AV, Masco HL, Rifkin SI, Acharya MK. Minimal-change disease and the nephrotic syndrome associated with lithium therapy. An Intern Med. 1980;92:70-2.

[5] Bear RA, Sugar L, Paul M. Nephrotic syndrome and renal failure secondary to lithium carbonate therapy. Can Med Assoc J. 1985;132:735-7.
[6] Santella RN, Rimmer JM, MacPherson BR. Focal segmental glomerulosclerosis in patients receiving lithium carbonate. Am J Med. 1988;84:951-4.

[7] Wood IK, Parmelee DX, Foreman JW. Lithium-induced nephrotic syndrome. Am J Psychiatry. 1989;146:84-7.

[8] Tam VK, Green J, Schwieger J, Cohen AH. Nephrotic syndrome and renal insufficiency associated with lithium therapy. Am J Kidney Dis. 1996;27:715-20.

[9] Gill DS, Chhetri M, Milne JR. Nephrotic syndrome associated with lithium therapy. Am J Psychiatry. 1997;154:1318-9.

[10] Schreiner A, Waldherr R, Rohmeiss P, Hewer W. Focal segmental glomerulosclerosis and lithium treatment. Am J Psychiatry. 2000;157:834.

[11] Herrero Mendoza MD, Caramelo C, Bellver Álvarez TM, López Cubero L. Síndrome nefrótico y tratamiento con litio. Med Clin (Barc). 2001;116:758-9.

[12] Naranjo CA, Busto U, Sellers EM, Sandor P, Ruiz I, Roberts EA, Janecek E, Domecq C, Greenblatt DJ. A method for estimating the probability of adverse drug reactions. Clin Pharmacol Ther. 1981;30:239-45.

[13] Phan L, Coulomb F, Boudon M, Gallois H, Kleinknecht D. Glomérulonéphrite extramembraneuse induite par le lithium. Néphrologie. 1991;12:185-7.

[14] Kala GK, Mogri M, Weber-Shrikant E, Springate JE. Lithium-induced membranous glomerulonephropathy in a pediatric patient. Pediatr Nephrol. 2009;24:2267-9 